\title{
Standards Development For Differential Scanning Calorimetry
}

Jane E. Callanan, Sandra A. Sullivan, and Dominic F. Vecchia

National Bureau of Standards Boulder, CO 80303
This article summarizes two studies made in preparation for standards development, by differential scanning calorimetry, for instruments such as scanning calorimeters, differential thermal analyzers, differential mechanical analyzers, and related thermal analysis devices. The first was an extensive study of the variability of differential scanning calorimeters when used for determining transition temperatures and enthalpies. The second was an evaluation of calibration procedures recom- mended by the American Society of Testing and Materials. These studies are described in detail in National Bureau of Standards Special Publication 260-99.

Key words: differential scanning calorimetry; fusion enthalpy; fusion temperature; pilot study; standards; thermal analysis; transition enthalpy; transition temperature.

Accepted: August 27, 1985.

\section{Introduction}

A need exists for a number of transition temperature standards for use with differential scanning calorimeters (DSCs), differential thermal analyzers (DTAs), and differential mechanical analyzers (DMAs). In addition, transition enthalpy standards are required for the DSC and DTA. Conventional calorimeters are absolute measuring instruments. Once such a calorimeter has been calibrated, the results obtained with it are thermodynamic values and remain so until the measuring system is altered. A repair to wiring within the measuring circuit, a change in the masses of various components of the calorimeter, or long-term aging and use effects

About the Authors: Jane E. Callanan and Sandra A. Sullivan are with the Center for Chemical Engineering in NBS' National Engineering Laboratory (NEL), while Dominic F. Vecchia is with NEL's Center for Applied Mathematics. necessitate recalibration. While the required time for the approach to equilibrium or shield behavior characteristics may depend on the nature of the specimen, the numerical results obtained do not as the system is an absolute measuring one.

On the other hand, most DSCs are not absolute measuring instruments; they require the use of standard substances to evaluate the correction factors, for both temperature and enthalpy, which must be applied in order to obtain corrected values for thermal properties.

The American Society for Testing and Materials (ASTM) recently issued revised calibration procedures for DSCs and DTAs [1,2]'. Preliminary evaluations of these procedures in our laboratory showed them to be promising, particularly if the melting points of the fusion standards were spaced at $50-60 \mathrm{~K}$ intervals.

\footnotetext{
${ }^{1}$ Figures in brackets indicate literature references.
} 
Existing temperature and enthalpy of fusion standards include six standard substances developed by the National Physical Laboratory (NPL), U.K. [3]. For these, temperatures have been determined in a triple-point cell; the enthalpies of fusion, in an adiabatic calorimeter.

A second group of substances, sponsored by the International Conference of Thermal Analysis (ICTA) in 1971, is available through the Office of Standard Reference Materials (OSRM) [4]. Theseare standards for temperature only; enthalpy values have not been reported. In addition, the uncertainty in the nominal values for the melting points cited for these latter substances is more than an order of magnitude greater than would be expected with more recent instrumentation. The cause of this uncertainty may lie in the substances themselves or it may be in techniques and instrumental factors associated with the initial measurements. Those measurements were intended for comparisons among instruments rather than for use as calibration standards.

A third set of reference materials, again only temperature standards, is also available from NPL. This consists of 10 organic compounds whose melting temperatures have been obtained in glass capillaries, at a heating rate of $2 \mathrm{~K} / \mathrm{min}$. The enthalpy of fusion standards have been selected from among these materials but were developed for different lots of material; thus the enthalpy values are not transferable.

For most reliable work with thermal analysis instruments it is desirable to have stancards available that cover a range of temperatures, enthalpies and materials, e.g., metals, organic solids, inorganic solids, liquids, polymers, etc. [5].

Experience in our laboratory led us to believe that we might be able to develop satisfactory temperature and enthalpy standard reference materials for DSCs with a DSC. Accordingly, a variability study and a calibration study to evaluate the feasibility of such work were designed and undertaken. A brief description of these studies, and the results obtained, is presented here. These results indicate that the proposed procedures do allow for certification of standards satisfactory for use with DSCs, DTAs, and other thermal analysis instruments. A program of development of such standards has been undertaken at NBS-Boulder.

This article is intended to acquaint users of these instruments with the certification procedure. Full documentation and all data will be found in a cited NBS Special Publication [6].

\section{Variability Study}

To judge the suitability of a DSC for the development of standards, it is necessary to identify and evaluate the various sources of experimental variability in temperature and enthalpy measurements. In general, such variability could arise from the DSC, from the procedures and laboratory conditions for working with the DSC, or from inhomogeneity among specimens of the reference material. Studies to evaluate the effects of these sources of experimental error on the precision of DSC measurements are described in this section.

Three forms of indium (granules, rod, foil) were selected for this study. In the initial variability study, five specimens of each form were prepared and each specimen was run four times, remounting the specimens in the calorimeter between repeat measurements. The 20 measurements of temperature and enthalpy of transition on each form were obtained in random order over a long enough time period to include the effects of variations in line voltage, or other diurnal perturbations, on the results. Specimen masses varied from $0.93-3.49 \mathrm{mg}$; temperature and enthalpy of transition did not exhibit mass dependence in this range.

A statistical analysis was done (separately) on both temperature and enthalpy data for each form of indium. The analysis allowed for the possibility that large differences could exist between different specimens of the same indium form. Figures la and $1 \mathrm{~b}$ illustrate the specimen-to-specimen (and within specimen) variability that can be seen in the data for granular indium. Variance calculations on the indium data describe both the internal variability of the measurements on a given specimen and the variability between sets of measurements on different specimens.

Both between-specimen and within-specimen components of variance were calculated in each analysis. The between-specimen component of variance is primarily associated with material variability of a given indium form. Hence, it provides a quantitative measure of inhomogeneity in the specimens with respect to temperature or enthalpy of transition. The within-specimen variance is that which occurs in the absence of sample inhomogeneity or when measuring a single specimen repeatedly. It contains contributions to overall variability other than specimen-related ones. With the present experimental design, these include instrumental factors and the effects of remounting the specimens, of operating procedures, and of ambient conditions. 


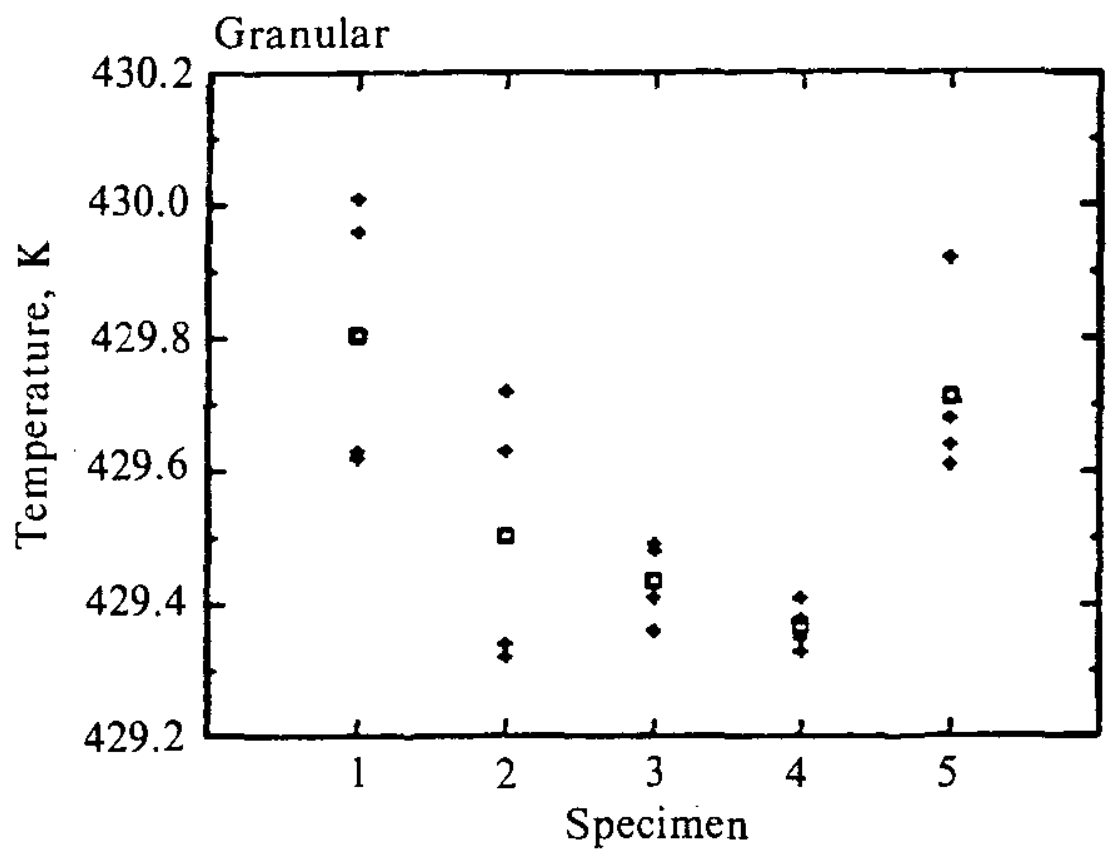

Figure 1a-Fusion temperature measurements for indium granules. ( $\mathbf{0}$ indicates specimen mean)

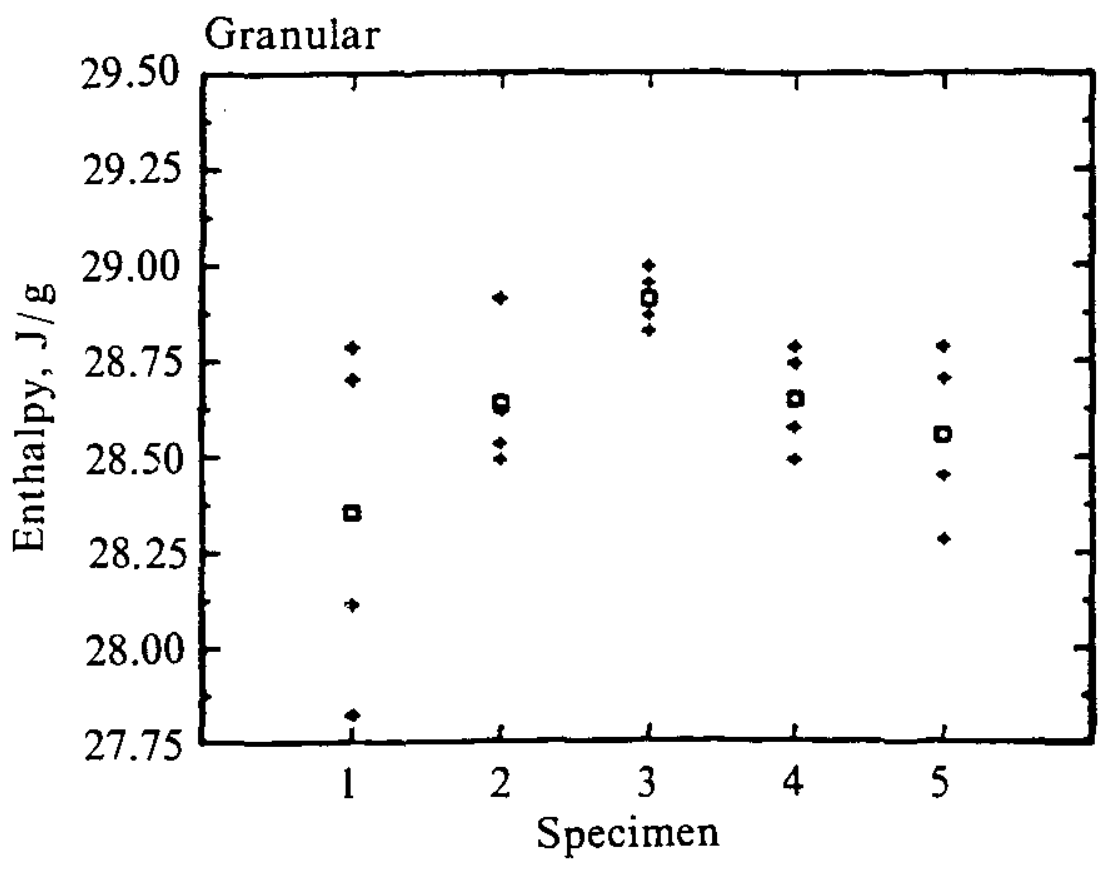

Figure 1b-Enthalpy measurements for indium granules. ( $\mathbf{a}$ indicates specimen mean)

For measurements on a given form of indium, the within-specimen component of variance, $S^{2}$, and between-specimen component of variance, $S_{b}{ }^{2}$, are calculated as follows:

and

$$
S_{\mathrm{b}}^{2}=\frac{1}{4}\left(4 S_{\mathrm{A}}^{2}-S^{2}\right)
$$$$
S^{2}=\frac{1}{15} \sum_{i=1}^{S} \sum_{j=1}^{4}\left(X_{i j}-\bar{X}_{i}\right)^{2}
$$

where

$$
S_{\mathrm{A}}^{2}=\frac{1}{4} \sum_{i=1}^{5}\left(\bar{X}_{i}-\bar{X}\right)^{2}
$$

In these formulae, $X_{i j}$ denotes the $j$-th measured temperature (or enthalpy) on specimen $i, \bar{X}_{i}$ is the average of four values on specimen $i$, and $\bar{X}_{i}$ is the grand average of all 20 values from 5 specimens. 
Note that $S^{2}$ (15 degrees of freedom) is, in fact, the pooled within-specimen variance, commonly used by chemists,

$$
S^{2}=\frac{1}{5} \sum_{i=1}^{5} S_{i}^{2}
$$

where $S_{i}{ }^{2}$ is the usual estimated variance of the four repeat measurements on the $i$-th specimen. Thus, $S^{2}$ is a proper estimate for the (internal) variability of repeat measurements on a given specimen when there may be large differences between different specimens of an indium form. Also, $S_{\mathrm{A}}{ }^{2}$ is the estimated variance of specimen means, and in effect, reflects both between specimen variability and within specimen experimental errors. The formula for the between specimen component of variability, $S_{\mathrm{b}}{ }^{2}$, is obtained from standard statistical theory [7].

To obtain a realistic estimate of the standard error in the grand average $\bar{X}$, of all measurements on a given form of indium, the within specimen and between specimen components of variance are combined in the formula:

$$
S^{2}(\bar{X})=\frac{S^{2}+4 S_{\mathrm{b}}{ }^{2}}{20}
$$

The average value, $\bar{X}$, is an estimate of the average melting temperature (or enthalpy) of all specimens of a given form of indium, and eq (2) is the correct estimate of the standard error that should be used to assess the uncertainty in the average value. There are four degrees of freedom for $S^{2}(\bar{X})$ in this analysis [7]. The average value, with its associated uncertainty, defines the limits within which investi- gators using the same material should expect their values to fall.

Estimates from this study of the average melting temperature and enthalpy of transition for each form of indium are given in table 1 and 2, respectively. Standard errors for these values were calculated using eq (2). Estimated standard deviations of repeat measurements on a single indium specimen are also given in the tables. These reported values were computed from the pooled within specimen variability using eq (1), because the usual formula for the standard deviation of all 20 measurements is inappropriate when there may be differences between sets of values from different specimens.

Estimates of the between-specimen component of variability of indium forms are given in table 3 . A statistical test of the hypothesis of no specimento-specimen variability was computed in each analysis. Significance levels for the tests are reported in table 3. The levels that were attained show evidence of variation in melting temperature among specimens of granular indium, but no evidence of significant variation for either rod or foil. However, all three forms of indium exhibited possible inhomogeneity among specimens with respect to enthalpies of transition. Reasons for the apparent difference in properties of indium with respect to the two measured quantities are currently being investigated.

Because the initial study could not distinguish remount variability from variation due to the instru ment itself, an additional six specimens (two of each form) were run three times in succession, with

Table 1. Mean temperatures obtained in indium standards study!

\begin{tabular}{lccccc}
\hline \hline & $\begin{array}{c}\text { Mean } \\
(\mathrm{K})\end{array}$ & $\begin{array}{c}\text { Standard } \\
\text { Error } \\
(\mathrm{K})\end{array}$ & $\begin{array}{c}\text { Degrees } \\
\text { of } \\
\text { Freedom }\end{array}$ & $\begin{array}{c}\text { Standard Deviation } \\
\text { Among Repeats } \\
(\mathrm{K})\end{array}$ & $\begin{array}{c}\text { Degrees } \\
\text { of } \\
\text { Freedom }\end{array}$ \\
\hline Granular & 429.565 & 0.083 & 4 & 0.148 & 15 \\
Rod & 429.509 & 0.038 & 4 & 0.168 & 15 \\
Foil & 429.602 & 0.038 & 4 & 0.170 & 15 \\
\hline
\end{tabular}

'See footnote, table 1.

Table 2. Mean enthalpies obtained in indium standards study'.

\begin{tabular}{lccccc}
\hline & $\begin{array}{c}\text { Mean } \\
(\mathrm{J} / \mathrm{g})\end{array}$ & $\begin{array}{c}\text { Standard } \\
\text { Error } \\
(\mathrm{J} / \mathrm{g})\end{array}$ & $\begin{array}{c}\text { Degrees } \\
\text { of } \\
\text { Freedom }\end{array}$ & $\begin{array}{c}\text { Standard Deviation } \\
\text { Among Repeats } \\
(\mathrm{J} / \mathrm{g})\end{array}$ & $\begin{array}{c}\text { Degrees } \\
\text { of } \\
\text { Freedom }\end{array}$ \\
\hline Granular & 28.623 & 0.089 & 4 & 0.256 & 15 \\
Rod & 28.761 & 0.105 & 4 & 0.270 & 15 \\
Foil & 28.936 & 0.055 & 4 & 0.148 & 15 \\
\hline
\end{tabular}

\footnotetext{
'See footnote, table 1.
} 
Table 3. Estimated specimen-to-specimen variation.

\begin{tabular}{lcccc}
\hline & \multicolumn{2}{c}{ Temperature } & & Enthalpy \\
& $\begin{array}{c}\text { Specimen- } \\
\text { to-Specimen } \\
S_{\mathrm{b}}{ }^{2}\end{array}$ & $\begin{array}{c}\text { Significance } \\
\text { Level, Test } \\
\sigma_{\mathrm{b}}=0\end{array}$ & $\begin{array}{c}\text { Specimen- } \\
\text { to-Specimen } \\
S^{2}\end{array}$ & $\begin{array}{c}\text { Significance } \\
\text { Level, Test } \\
\sigma_{\mathrm{b}}=0\end{array}$ \\
\hline Granular & $(0.171)^{2}$ & 0.0034 & $(0.153)^{2}$ & 0.0937 \\
Rod & 0 & 0.5419 & $(0.193)^{2}$ & 0.0502 \\
Foil & 0 & 0.7977 & $(0.097)^{2}$ & 0.0692 \\
\hline
\end{tabular}

no operator interference, to quantify the lattersource of experimental error. Additional studies allowed the evaluation of weighing error variation, effects of the computer analysis routine, and differences between operators. Estimates of the contributions to overall variability in DSC measurements from various sources of experimental variability are shown in table 4. (Only results for foil are given because the previous analysis shows foil measurements to be the preferred (i.e., less variable) form of indium for standards development.)

The results in table 4 were calculated in an ad hoc fashion from the combined results of the initial study and the additional data that were obtained later. Variation between operators is omitted from the table because operator differences were negligible. Errors introduced by the analytic procedure and specimen differences were negligible for temperature. They were not for enthalpy; in fact, specimen differences were the second most important component of the variance of the enthalpy. Variability introduced by the instrument itself, without contribution from remounting the specimen, was small in both instances. The most serious contributions to the variance of both temperature and enthalpy occurred as a result of replacing the specimens in the calorimeter.

\section{Calibration Study}

The second part of the study provided an evaluation of the temperature and enthalpy calibration

Table 4. Contributions to experimental variability (foil).

\begin{tabular}{lcc}
\hline & $\begin{array}{c}\text { Temperature } \\
\text { Estimated } \\
\text { Variance } \\
\text { Component } \\
{\left[\mathrm{K}^{2}\right]}\end{array}$ & $\begin{array}{c}\text { Enthalpy } \\
\text { Estimated } \\
\text { Variance } \\
\text { Component } \\
{\left[(\mathrm{J} / \mathrm{g})^{2}\right]}\end{array}$ \\
\hline Specimen & 0 & $(0.097)^{2}$ \\
Analytic Procedure & 0 & $(0.055)^{2}$ \\
Instrument & $(0.016)^{2}$ & $(0.023) 2$ \\
Remount & $(0.169)^{2}$ & $(0.136)^{2}$ \\
Total & $(0.170)^{2}$ & $(0.177)^{2}$ \\
\hline
\end{tabular}

procedures for DSC which have been recommended recently by the ASTM $[1,2]$.

For temperature, the fusion temperatures for two well-characterized materials which bracket the temperature of interest were determined. The calculation of the observed transition temperature was obtained from

$$
T=(T O \times S)+I
$$

where $T$ is the corrected specimen temperature, $T \mathrm{O}$ is the observed temperature, $S$ is the slope and $I$, the intercept. The slope and intercept are calculated from eqs (5) and (6).

$$
\begin{gathered}
S=\left(T S_{1}-T S_{2}\right) /\left(T \mathrm{O}_{1}-T \mathrm{O}_{2}\right) \\
I=\left[\left(T \mathrm{O}_{1} \times T S_{2}\right)-\left(T S_{1} \times T \mathrm{O}_{2}\right)\right] /\left(T \mathrm{O}_{1}-T \mathrm{O}_{2}\right) .
\end{gathered}
$$

The $T S_{i}$ are the literature values for the transition temperatures.

The associated enthalpies are corrected as follows. The transition enthalpy of a reference standard, usually indium, is measured and a calibration factor for the instrument determined at the transition temperature of this substance. The enthalpy correction is extended to other temperatures through use of a ratio of the heat capacity of a second standard, determined by the enthalpic method, at the reference temperature and at the temperature of interest. Sapphire is usually chosen for the second, heat capacity, standard because of its well-documented specific heat. The calibration coefficient $(E)$ is obtained from the ratio of the literature value of the enthalpy of transition $\left(\Delta H_{\text {lit }}\right)$ to the observed value $\left(\Delta H_{o b s}\right)$,

$$
E=\left(\Delta H_{\text {lit }}\right) /\left(\Delta H_{\text {obs }}\right)
$$

Then the corrected enthalpy of fusion $\Delta H$ (corr) of measured specimens is obtained from

$$
\Delta H(\text { corr })=E \times \Delta H(\text { meas }),
$$


where $\Delta H$ (meas) is the measured enthalpy of fusion for that specimen.. For extension to other temperatures a correction factor, $F$, is obtained from the results for the heat capacity of sapphire, $C p$.

$$
F=C p(\mathrm{lit}) / C p \text { (obs) }
$$

The $\Delta H$ (corr) from eq (8) is multiplied by the ratio of the $F$-factor at the temperature of interest to the $F$-factor at the reference temperature. The $F$-factor normally changes somewhat with temperature; for that reason an $F$-factor specific to the temperature of interest is used.

The test materials selected were from the group of melting point standards certified by the NPL. The materials used and their reference temperatures are given in table 5 .

Table 5. Melting points of test materials (K).

\begin{tabular}{lcc}
\hline \hline Substance & NPL Certificate Values [8] & Literature Values \\
\hline Napthalene & $353.37 \pm 0.05^{*}$ & $353.37[9]$ \\
Acetanilide & $387.51 \pm 0.05$ & $387.51[9]$ \\
Diphenylacetic & $420.41 \pm 0.05$ & $420.41[9]$ \\
$\quad$ Acid & & \\
Anisic Acid & $456.45 \pm 0.2$ & $456.14[10]$ \\
2-Chloroanthra- & $482.75 \pm 0.3$ & $482.20[10]$ \\
$\quad$ quinone & & \\
\hline
\end{tabular}

* Uncertainties represent one standard deviation.
The certificates for these materials indicate that these reference temperatures refer to a specific heating regime, heating at $2 \mathrm{~K} / \mathrm{min}$ with the specimen contained in a glass capillary tube. Where other definitive work has been done, the values are listed in column 3 of table 5 .

Five specimens of each of the five test materials were prepared; each was run four times. Randomly selected sets of the five substances were used and run in order of increasing temperature. Substances 1 and 3 were used, as bracketing substances, to calibrate the DSC and the transition properties of substance 2 were obtained. Then substances 2 and 4 were used for bracketing and the properties of substance 3 obtained. This procedure, followed through the selected list, would allow us to determine 1) whether the DSC would produce the accepted temperatures satisfactorily and 2) what error is to be expected in various temperature ranges.

The results, as given in table 6 , separate the first run from the average of all four. Whether particular standards can be reused is always a question. Table 6 shows that, for the substances used here, differences between the first and the subsequent runs are of no practical significance. In those instances in which sample deterioration occurs, usually manifested by peak distortion, standard specimens cannot be reused.

Table 6. Summary of transition temperatures and enthalpies.

\begin{tabular}{|c|c|c|c|}
\hline & Substance & $\begin{array}{c}\text { Transition } \\
\text { Temperature (K) }\end{array}$ & $\begin{array}{c}\text { Enthalpy of } \\
\text { Transition }(\mathrm{J} / \mathrm{g})\end{array}$ \\
\hline \multicolumn{4}{|l|}{ INITIAL RUN } \\
\hline & Naphthalene & & $149.35 \pm 0.85 t$ \\
\hline & Acetanilide & $387.35 \pm 0.28 \uparrow$ & $163.65 \pm 1.26$ \\
\hline & Diphenylacetic Acid & $420.22 \pm 0.16$ & $148.67 \pm 1.32$ \\
\hline & Anisic Acid & $456.18 \pm 0.41$ & $195.49 \pm 0.76$ \\
\hline & 2-Chloroanthraquinone & & $148.15 \pm 0.76$ \\
\hline
\end{tabular}

ALL RUNS

Naphthalene
Acetanilide
Diphenylacetic Acid
Anisic Acid
2-Chloroanthraquinone

$387.38 \pm 0.37$

$420.23 \pm 0.39$

$456.50 \pm 0.43$

$149.01 \pm 1.18$

$163.40 \pm 1.34$

$148.16 \pm 1.28$

$195.31 \pm 1.53$

$146.81 \pm 1.20$

\section{LITERATURE}

353.37 [9]
387.51 [9]
420.41 [9]
$456.14[8]$
$482.20[8]$

$148.6[9]$

$160.2[9]$

147.3 [9]

$207.91[8]$

135.35 [8]

NOTE: Naphthalene and 2-chloroanthraquinone have no temperatures listed since they were the outer bracketing substances. 
The literature values quoted are for different lots of purified material from those used in this study or for results obtained by DSC in the early 1970s. Both DSC instruments and measuring techniques have improved greatly in the intervening years. Therefore, these results quoted here as literature values do not provide reliable criteria for comparison, but none more reliable are available currently.

\section{Summary}

The work reported here demonstrates that new standard reference materials for thermoanalytical instruments can be developed using the ASTM Recommended Practices [1,2]. The certified values for these materials will carry uncertainties in temperature an order of magnitude less than the ICTA standards presently in use. In addition they will be certified simultaneously for transition enthalpies. The best results are obtained when like substances are used for the calibrations and when the temperature difference between calibrants does not exceed $50 \mathrm{~K}$. No blanket statement can be made about reusing standard specimens. If the fusion curves are not distorted, the specimen should be used again; if the curves are misshapen the specimen may not be reused.

It is anticipated that some of the materials that will be developed as standards, e.g., powders or crystals, will be readily homogenized. For these materials statistics which are appropriate for homogeneous materials, ungrouped data, will be applied. For materials which exhibit some inhomogeneity, as did some of the metallic samples used in this study, a statistical model which can separate the effects of inhomogeneity from other factors will be applied.

The results of this study, summarized here, make the use of a DSC for the development of fusion standards for the broad class of thermal analytical instruments creditable. A long-range program for the development of such standards is underway in this laboratory.

The authors thank Keith R. Eberhardt of the Center of Applied Mathematics, National Bureau of Standards-Gaithersburg for helpful advice and discussion concerning this study and the manuscript.

\section{References}

[1] ASTM Standard Practice for temperature calibration of differential scanning calorimeters and differential thermal analyzers E967-83. Annual Book of Standards. 14.02: 782787 (1984).

[2] ASTM Standard Practice for heat flow calibration of differential scanning calorimeters E968-83. Annual Book of Standards. 14.02: 788-794 (1984).

[3] NPL Certified Reference Materials and Transfer Standards. National Physical Laboratory, Teddington, U.K. (1982 July).

[4] Office of Standard Reference Materials, National Bureau of Standards, Gaithersburg, MD 20899.

[5] Flynn, J. H., Theory of differential scanning calorimetry coupling of electronic and thermal steps, in Analytical Calorimetry, Vol. 3, R. S. Porter and J. F. Johnson, eds. New York, NY: Plenum Press, 17-44 (1974).

[6] Callanan, J. E.; S. A. Sullivan, and D. F. Vecchia, Feasibility study for the development of standards using differential scanning calorimetry. National Bureau of Standards (U.S.) Spec. Publ. 260-99 (1985 May).

[7] Box, G. E. P.; W. G. Hunter, and J. S. Hunter, Statistics for Experimenters, New York: John Wiley, 571-583 (1978).

[8] NPL Certificate of measurement CRM No. M14-11. Set of ten melting point standards. National Physical Laboratory, Teddington, U.K. (1980 November).

[9] Andon, R. J. L., and J. E. Connett, Calibrants for thermal analysis. Measurement of their enthalpies of fusion by adiabatic calorimetry. Thermochimica Acta 42: 241-247 (1980).

[10] Pella, E., and M. Nebuloni, Temperature measurements with a differential calorimeter. Journal of Therm. Anal. 3: 229-246 (1971). 